\title{
Exciton Confinement in InGaN/GaN Cylindrical Quantum Wires
}

\author{
E. W. S. Caetano*, V. N. Freire*, G. A. Farias*, and E. F. da Silva Jr. ${ }^{\dagger}$ \\ * Departamento de Física, Universidade Federal do Ceará, Centro de Ciências Exatas, \\ Campus do Pici, Caixa Postal 6030, 60455-900, Fortaleza, CE, Brazil \\ † Departamento de Física, Universidade Federal de Pernambuco, \\ Cidade Universitária, 50670-901, Recife, PE, Brazil
}

Received on 31 March, 2003

\begin{abstract}
We investigate the confined exciton properties in InGaN/GaN cylindrical quantum wires. We have solved numerically Schrödinger-like equations in the effective mass approximation and have found the energy levels and wavefunctions for confined electrons and holes, using these results to calculate variationally the confined exciton energy. A comparison was made between graded and abrupt quantum wires, showing that the existence of smooth interfaces enhances the confined exciton energy if the wire radius is smaller than $50 \AA$. We conclude that the existence of graded interfaces must be considered for a better description of the exciton based optical properties of InGaN/GaN quantum wires.
\end{abstract}

\section{Introduction}

Blue and ultraviolet light emitting diodes (LEDs) and blue laser diodes (LDs) have been developed recently using InGaN quantum wells (QWs) as the active layers [1,2]. However, by comparing the density of states of 3D, 2D, and 1D systems, it is clear that a device operating in one dimension has potential to be more efficient than $3 \mathrm{D}$ and $2 \mathrm{D}$ dimensional devices. This has motivated extensive research in nanowire technology, including the aim of understanding fundamental 1D physics and material proceses.

Recently, Han et al. [3] reported the fabrication of GaN nanorods, while Li et al. [4] have grown nanoporous GaN structures from crystalline $\mathrm{GaN}$ on conducting $\mathrm{SiC}$ substrate using etching in $\mathrm{HF} / \mathrm{H}_{2} \mathrm{O}_{2}$. These structures have shown a blueshifted emission correlated with the development of highly anisotropic wire-like structures in the morphology, with the higher energy cathodoluminescence band arising from the portion of the structure with the smallest feature sizes. Krost et al. [5] have found pure metallic indium in thick (300 nm) MOCVD-grown InGaN layers incorporated in a wire-like manner along the growth direction. Such indium wires could act as highly conducting channels harmful for electronic and optoelectronic applications.

We investigate the ground state confined exciton properties. In cylindrical wurtzite $\operatorname{In}_{0.2} \mathrm{Ga}_{0.8} \mathrm{~N} / \mathrm{GaN}$ quantum wires. Since interfaces $1-1.5 \mathrm{~nm}$ thick in $\mathrm{Al}$ $\mathrm{GaN} / \mathrm{InGaN} / \mathrm{GaN}$ heterostructures were demonstrated $[6,7]$ we take into account their existence.

\section{The numerical method}

The $\mathrm{In}_{0.2} \mathrm{Ga}_{0.8} \mathrm{~N} / \mathrm{GaN}$ cylindrical quantum wires are modeled as follows. The InN molar fraction depends on the distance from the wire axis according to:

$$
\chi(r)=0.1\left\{1-\operatorname{erf}\left(\frac{2(r-R)+\sigma}{\sigma / 2}\right)\right\},
$$

where $\mathbf{R}$ is the quantum wire radius and $\sigma$ is the graded interface thickness. The effective mass approximation is adopted, and the BenDaniel-Duke kinetic energy operator is part of the exciton Hamiltonian:

$$
\begin{aligned}
& \widehat{\mathrm{H}}=E_{g}(\chi=0.2) \underbrace{-\frac{\hbar^{2}}{2} \nabla_{e}\left(\frac{1}{m_{e}\left(r_{e}\right)}\right) \nabla_{e}+V_{e}\left(r_{e}\right)}_{\widehat{\mathrm{H}}_{e}} \\
& \underbrace{-\frac{\hbar^{2}}{2} \nabla_{h}\left(\frac{1}{m_{h}\left(r_{h}\right)}\right) \nabla_{h}+V_{h}\left(r_{h}\right)}_{\widehat{\mathrm{H}}_{h}} \underbrace{-\frac{e^{2}}{4 \pi \varepsilon \varepsilon_{0}\left|\mathbf{r}_{\mathbf{e}}-\mathbf{r}_{\mathbf{h}}\right|}}_{\widehat{\mathrm{V}}_{e h}},
\end{aligned}
$$

where $m_{e}\left(m_{h}\right)$ and $V_{e}\left(V_{h}\right)$ are, respectively, the electron (heavy hole) effective mass and confinement potential. The effective masses are isotropic, calculated by averaging in the parallel and perpendicular directions to the [0001] growth axis; $\varepsilon$ is the $\operatorname{In}_{0.2} \mathrm{Ga}_{0.8} \mathrm{~N}$ dielectric constant. The confinement potentials, on the other hand, depend on the band offsets, taken here to be $Q_{e}=0.7, Q_{h}=1-Q_{e}=0.3$; the bowing parameter is assumed to be $b=2.6 \mathrm{eV}$ [8]. The parameters for the $\operatorname{In}_{0.2} \mathrm{Ga}_{0.8} \mathrm{~N}$ alloy were obtained by interpolation of the InN and GaN presented in Table I.

The variational wavefunction we used for the exciton ground state is:

$$
\psi_{e h}=\psi_{e}\left(r_{e}\right) \psi_{h}\left(r_{h}\right) e^{-\frac{\sqrt{r_{e}^{2}+r_{h}^{2}-2 \beta r_{e} r_{h}}}{\lambda}} e^{-\frac{z_{e}^{2}+z_{h}^{2}}{\lambda}},
$$


Table I - InN and GaN data [8,9].

\begin{tabular}{ccc}
\hline & InN & GaN \\
\hline $\boldsymbol{E}_{g}(\mathbf{e V})$ & 2.050 & 3.520 \\
\hline $\boldsymbol{m}_{e} / \boldsymbol{m}_{o}$ & 0.125 & 0.206 \\
\hline $\boldsymbol{m}_{h} / \boldsymbol{m}_{o}$ & 1.547 & 1.081 \\
\hline$\varepsilon$ & 14.60 & 10.30
\end{tabular}

where $z_{i}(i=e, h)$ is the carrier coordinate measured along the quantum wire axis, which is the growth direction [0001]; $\psi_{i}$ is the ground state solution of $H_{i} \psi_{i}=E_{i} \psi_{i} . \lambda$ and $\beta$ are two variational parameters associated to the radius and symmetry of the ground state exciton, respectively. The exciton grounde state energy is obtained variationally through the following expression:

$$
E_{E X C}^{0}=\min _{\lambda, \beta} \frac{\left\langle\psi_{e h}|\widehat{\mathrm{H}}| \psi_{e h}\right\rangle}{\left\langle\psi_{e h} \mid \psi_{e h}\right\rangle},
$$

where $E_{B}^{0}=E_{E X C}^{0}-E_{g}(\chi=0.2)-E_{e}-E_{h}$ is the exciton binding energy.

\section{The confined exciton}

Figure 1 shows the binding (top) and total exciton (bottom) energies in graded $\mathrm{In}_{0.2} \mathrm{Ga}_{0.8} \mathrm{~N} / \mathrm{GaN}$ cylindrical quantum wires for interface thicknesses ranging from 0 (abrupt quantum wire) to $15 \AA$. The exciton binding energy in the case of an abrupt wire varies from $55 \mathrm{meV}(\mathrm{R}=20 \AA)$ to 24 $\mathrm{meV}(\mathrm{R}=80 \AA)$. When a graded interface $10 \AA(15 \AA)$ is considered, the binding energy increases by as much as $8 \mathrm{meV}$ (10 meV) when $\mathrm{R}=20 \AA$. The total exciton energy shifts to lower energies as the quantum wire radius increases. When the interface is sharp (abrupt), it varies from $2.97 \mathrm{eV}(\mathrm{R}=20$ $\AA$ ) to $2.81 \mathrm{eV}$, which corresponds to the blue color in the visible spectrum. Taking into account a graded interface 10 $\AA$ thick, the exciton energy is shifted towards higher energies. For a $20 \AA$ wire radius, the exciton energy is $3.08 \mathrm{eV}$ (violet).

The exciton energy blue shift due to the existence of a graded interface in the quantum wire is given by $\Delta E_{E X C}(R, \sigma)=E_{E X C}^{0}(R, \sigma)-E_{E X C}^{0}(R, \sigma=0)$. Figure 2 depicts $\Delta E_{E X C}(R, \sigma)$ for $\sigma=5 \AA, 10 \AA, 15 \AA$. From this figure it is clear that graded interfaces represent an important factor to be taken into account if the wire radius is smaller than $50 \AA$. Even for an interface thickness of only $5 \AA$, the energy blue shift for $\mathrm{R}=20 \AA$ is $\sim 50 \mathrm{meV}$. Effects related to the existence of graded interfaces can be neglected only for quantum wires with radius larger than $50 \AA$.

\section{Conclusion}

Remarkable interface related blue shifts on the exciton total energy in $\mathrm{In}_{0.2} \mathrm{Ga}_{0.8} \mathrm{~N} / \mathrm{GaN}$ cylindrical quantum wires was shown to occur. These blue shifts are more pronounced for
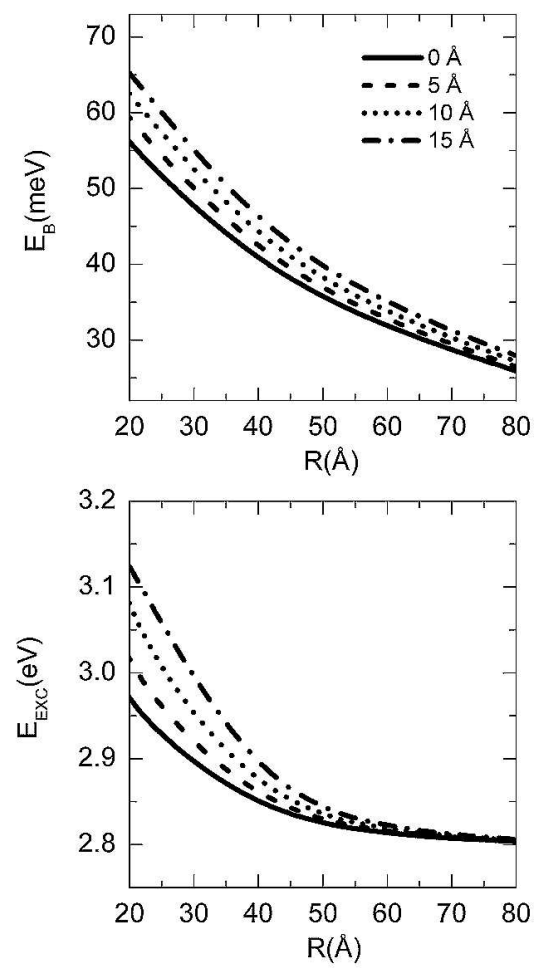

Figure 1. Shows the ground state binding (top) and total (bottom) ground state e-h confined exciton energy in $\operatorname{In}_{0.2} \mathrm{Ga}_{0.8} \mathrm{~N} / \mathrm{GaN}$ quantum wires with interface thickness $\sigma=0 \AA$ (solid), $5 \AA$ (dashed), $10 \AA$ (dotted), and $15 \AA$ (dotted dashed).

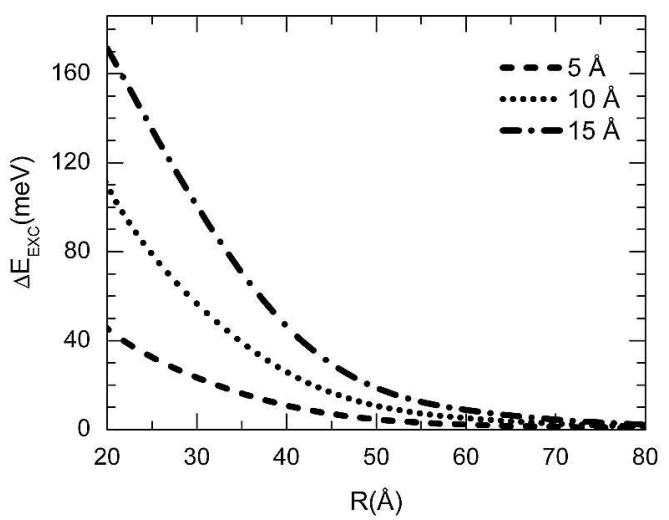

Figure 2. Exciton energy blue shift $\Delta E_{E X C}(R, \sigma)$ in $\mathrm{In}_{0.2} \mathrm{Ga}_{0.8} \mathrm{~N} / \mathrm{GaN}$ quantum wires with interface thickness $5 \AA$ (dashed), $10 \AA$ (dotted), and $15 \AA$ (dotted dashed).

quantum wires with radius smaller than $50 \AA$, reaching values between $50 \mathrm{meV}$ and $170 \mathrm{meV}$ depending on the interface thickness and the wire radius. The enhancement of the confined exciton energy significantly enlarges the region of the visible spectrum spanned and must be taken into account for a better description of the optoelectronic properties of InGaN/GaN quantum wires.

A drawback of our calculations is that we have not taken into account the existence of an internal electric field due to 
spontaneous and piezoelectric polarizations in wurtzite nitrides, which are highly important $[8,9]$. However, work is in development to include these fields in the confined exciton calculations in III-nitride based wires.

\section{Acknowledgement}

E. W. S. Caetano would like to acknowledge the graduate fellowship at the Physics Department of the Universidade Federal do Ceará received from CAPES during the realization of this research. V. N. F., G. A. F. and E. F. S. Jr. would like to acknowledge the financial support received from the Science Funding Agency of the Ceará (FUNCAP) and of the Pernambuco (FACEPE) states in Brazil, and the Brazilian National Research Council (CNPq) through the grant NanoSemiMat Project N. 550.015/01-9.

\section{References}

[1] S. Nakamura, Science 281, 956 (1998).
[2] S. Nakamura and G. Fasol, The Blue Laser Diode (Springer, Berlin, 1997).

[3] W. Han, S. Fan, Q. Li, and Y. Hu, Science 277, 1287 (1997).

[4] X. Li, Y.-W. Kim, P. W. Bohn, and I. Adesida, Appl. Phys. Lett. 80, 980 (2002).

[5] A. Krosta, J. Bläsing, H. Protzmann, M. Lünenbürger, and M. Heuken, Appl. Phys. Lett. 76, 1395 (2000).

[6] C. Kisielowski, Z. Liliental-Weber, and S. Nakamura, Jpn. J. Appl. Phys. 36, 6932 (1997).

[7] T. Shirasawa, N. Mochida, A. Inoue, T. Honda, T. Sakaguchi, F. Koyama, and K. Iga, J. Crystal Growth 189/190, 124 (1998).

[8] E. W. S. Caetano, V. N. Freire, G. A. Farias, and E. F. da Silva Jr., Physica E 13, 1106-1110 (2002).

[9] F. Bernardini, V. Fiorentini, and D. Vanderbilt, Phys. Rev. B 56, R10024 (1997). 